\title{
Factores protectores y los estilos de crianza: un modelo bioecológico
}

Protective factors and parenting styles: A bio-ecological model

\author{
José Concepción Gaxiola Romero \\ Martha Frías Armenta \\ Universidad de Sonora \\ Aurelio José Figueredo \\ University of Arizona
}

\section{Resumen}

El objetivo del estudio fue evaluar, empleando la teoría bioecológica de Bronfenbrenner, los efectos de factores protectores en la crianza. 183 madres con historia de abuso, habitantes de la ciudad de Hermosillo, Sonora, México, aceptaron participar en la investigación. Los datos fueron analizados empleando ecuaciones estructurales. Los resultados muestran que el factor K modula el efecto de los factores protectores. Éstos y los de riesgo se encontraron tanto en el exosistema, como en el microsistema y en el ontosistema. El factor protector predijo los estilos de crianza, amortiguando los efectos de las variables de riesgo como la inseguridad del vecindario, la violencia de pareja y la depresión.

\section{Abstract}

The aim of this study was to evaluate the effects of protective factors on parenting styles, based on Bronfenbrenner's bio-ecological theory. One-hundred-and-eighty-three mothers living in Hermosillo, Sonora, Mexico, with child abuse history, accepted to participate in the research. The data were analyzed using structural equation modeling. Results showed that $\mathrm{K}$ factor modulates the effect of protective factors. Protective and risk factors were found in the exosistem, microsistem and ontosistem. Protective factor predicted parenting styles, buffering the effects of risk factors like neighborhood insecurity, partner violence, and depression. 


\section{Introducción}

Los estilos de crianza implican valores y metas de socialización de los hijos, las prácticas educativas específicas que utilizan los padres y las actitudes que expresan hacia los hijos (Darling y Steinberg, 1993). Baumrind (1967; 1983; 1991) propuso una caracterización de estos basándose en el tipo de control ejercido por los padres hacia los hijos. De este análisis resultaron tres tipos de estilos cualitativamente diferentes: los padres autoritativos, los autoritarios y los permisivos.

De acuerdo con Baumrind (1991) los padres autoritativos agrupan un conjunto de características que abarcan apoyo emocional, posibilidad de autonomía y comunicación bidireccional, lo cual permite a niños y adolescentes desarrollar competencia instrumental, caracterizada por cooperación con adultos y compañeros, independencia responsable y madurez psicosocial.

El estilo autoritario está asociado con el empleo del castigo físico y verbal como método disciplinario de los hijos (Frías-Armenta, López-Escobar y Díaz-Méndez, 2003). El castigo corporal aplicado a los hijos tiene relación con la presencia de comportamientos negativos, tales como conducta antisocial y violenta, sentimientos de miedo, ansiedad y enojo, sentimientos depresivos y con la reproducción en la etapa adulta de las prácticas agresivas con los propios hijos y el abuso infantil (Frías, 2002; Gershoff, 2002).

Los padres pasivos pueden ser padres indulgentes que son bajos en demandas y altos en responsividad (reforzamiento), o bien, como señalan Maccoby y Martin (1983), pueden ser bajos en demandas y bajos en responsividad. El bajo envolvimiento de los padres, definido como el grado de interés en las actividades de los hijos durante la niñez (una variable relacionada con el estilo pasivo), está asociado con los logros escolares bajos en niños de primaria (Englund, Luckner, Whaley y Egeland, 2004), y con la pobre salud psicológica de las mujeres en la edad adulta (Flouri, 2005).
Las consecuencias psicosociales en los niño(a)s relacionadas con los estilos de crianza, establecen la necesidad de realizar investigaciones que identifiquen los factores que pueden afectarlos.

\section{El modelo bioecológico}

Bronfenbrenner (1979) desarrolló un modelo ecológico que está basado en círculos concéntricos de mutua influencia divididos en tres contextos principales: el macrosistema, el exosistema y el microsistema. El macrosistema es el nivel más amplio e incluye las formas de organización social, las creencias culturales y los estilos de vida que prevalecen en una cultura o subcultura particular. El segundo nivel es el exosistema y está conformado por el sistema de relaciones enmarcadas por las instituciones que median entre la cultura y el nivel individual como la escuela, los organismos judiciales, las instituciones de seguridad y la Iglesia. El tercer nivel lo forma el microsistema, compuesto por las relaciones más cercanas de las personas, el cual está caracterizado fundamentalmente por las relaciones familiares, y además, está conformado por variables individuales. Belsky (1980) agregó el nivel del ontosistema para incluir los factores individuales (originalmente del microsistema). El modelo ecológico original de Bronfenbrenner (1979) fue modificado por Bronfenbrenner y Ceci (1994) agregando al paradigma ecológico el elemento biológico, llamándolo a partir de entonces teoría bioecológica del desarrollo humano, la cual enfatiza ciertos mecanismos de interacción del organismo con el ambiente nombrados en la teoría "factores proximales", cuya importancia radica en que, a través de ellos, los genotipos (producto de la herencia) son transformados en fenotipos (la expresión comportamental del organismo). La teoría bioecológica establece que el ser humano es un ente activo involucrado en un proceso continuo de cambios de sus características biopsicológicas. Dicho proceso es modulado tanto por las experiencias de los sujetos con los diversos contextos en donde se desenvuelve, así 
como por las influencias genéticas manifestadas en dichos intercambios. Al igual que en la propuesta inicial de la teoría ecológica, las influencias ambientales van desde las directas establecidas en los contextos inmediatos de los sujetos (micro y exosistema), denominadas procesos proximales, hasta las remotas e indirectas constituidas por contextos más globales (macrosistema) llamadas procesos distales. En la teoría bioecológica los procesos proximales constituyen las interacciones primarias del desarrollo humano. A partir de sus posibilidades analíticas, el modelo bioecológico puede servir de marco para el estudio de las variables protectoras o de riesgo relacionados con los estilos de crianza, al incluirse en el análisis variables de los diversos sistemas, y se agrega al menos, la influencia de una variable biológica. La crianza debido a que recibe la influencia de un conjunto de variables proximales y distales (Darling y Steinberg, 1993), requiere para su estudio de teorías que permitan un análisis holístico de las variables que la afectan.

Los factores protectores son aquellas condiciones o entornos capaces de favorecer el desarrollo de individuos o grupos y que pueden reducir los efectos de circunstancias desfavorables (Munist, Santos, Kotliarenco, Suárez, Infante y Grotberg, 1998). Estos factores funcionan, además, como defensas sobre los efectos negativos de las variables de alto riesgo (Cicchetti y Rogosch, 1997; Muller y Lemieux, 2000; Rutter, 1999; Rutter, 2007), y de acuerdo con la teoría ecológica de Bronfenbrenner (1979) pueden operar en todos los sistemas, por lo cual es necesario analizar cuáles son las variables que pueden ser más relevantes en cada uno de ellos.

En el macrosistema, el nivel socioeconómico alto puede constituirse como variable protectora. Los niveles altos de educación, el tener empleo, y la ausencia de presiones económicas pueden ser mecanismos protectores contra los estilos de crianza violentos (Carlson, McNutt y Choi, 2003).
En el nivel del exosistema pueden encontrarse como variables protectoras la cohesión y el apoyo social de vecinos. La cohesión implica el grado de confianza y de valores compartidos entre las personas que viven en el vecindario (Silk, Sessa, Morris, Steinberg y Avenevoli, 2004). Las investigaciones sugieren que las habilidades de los padres para el empleo de estrategias de crianza exitosas, dependen en parte, de los niveles de apoyo social brindados y el estrés experimentado fuera de casa, en los contextos que rodean a la familia (Bowen, Bowen y Ware, 2002; Korbin, 2003).

A nivel del microsistema puede incluirse como variable protectora el apoyo social de familiares y amigos y el de la pareja. En el caso de los padres y madres de familia, el apoyo social sirve para que ellos se sientan conectados a su comunidad y promueve que empleen estrategias disciplinarias no punitivas (Crouch y Behl, 2001; Garbarino y Kostelny, 1995). Por medio del apoyo social, los padres y madres pueden recibir soporte instrumental (por ejemplo dinero), emocional o información sobre estrategias de crianza (Crouch y Behl, 2001).

En el ámbito de la familia, el apoyo social entre las parejas afecta la relación entre los eventos traumáticos y su bienestar psicológico, al incrementar la satisfacción, el funcionamiento individual, bajar los niveles de estrés y disminuir los síntomas depresivos (Dehle, Larsen y Landers, 2001).

En el ontosistema pueden encontrarse las variables calidad de vida y autoestima. En un sentido amplio, la calidad de vida refiere a la satisfacción individual y a la felicidad en todas las dimensiones consideradas de importancia en la vida (Hyland, 1998). Algunas de las dimensiones del constructo de calidad de vida son predictores del ajuste psicológico; como por ejemplo, el estatus del empleo, la satisfacción laboral, la situación y la salud física (Louis y Zhao, 2002), las cuales pueden relacionarse con estrategias de crianza efectivas. 
La autoestima puede definirse como la suma de las creencias individuales, y el conocimiento acerca de los atributos y cualidades personales (Mann, Hosman, Schaalma y De Vries, 2004). La autoestima alta es un factor importante que contribuye a mejorar la salud y la calidad de vida (Zimerman, 2000); así mismo, es un predictor de la felicidad reportada (Furnham y Cheng, 2000), por lo cual es posible que afecte a los estilos de crianza efectivos.

El factor $\mathrm{K}$ alto de acuerdo con la teoría evolucionista, tiene que ver con comportamientos de alta inversión paterna, comportamiento prosocial y pensamiento a largo plazo (Figueredo et al., 2005), puede ser un mecanismo que afecta la influencia de los factores protectores contra las consecuencias adversas de los factores de riesgo. En la teoría bioecológica, el factor $\mathrm{K}$ constituye una variable psicobiológica del tipo proximal, que posiblemente afecte los estilos de crianza.

La inversión paterna, como uno de los indicadores del Factor K, se asocia con el compromiso y envolvimiento que los padres tienen con los hijos y constituye una variable integral de la crianza, debido a la dependencia de los niños con respecto a los adultos que los rodean (Darling y Steinberg, 1993).

Con respecto a los factores de riesgo, éstos constituyen cualquier característica o cualidad de una persona o comunidad que va unida a una elevada probabilidad de dañar la salud (Munist et al., 1998). Los factores de riesgo no causan directamente consecuencias negativas, sino que establecen las circunstancias que las probabilizan (Luthar, Cicchetti y Becker, 2000; Tussaie y Dyer, 2004).

Como variable de riesgo en el exosistema pueden incluirse las características de inseguridad del vecindario. Estas representan un riesgo indirecto para la aplicación de estrategias de crianza violentas, debido a que afectan primeramente el bienestar general de las familias (DuMont, Widom y Czaja, 2007). La vio- lencia en la pareja se ubicará en el modelo, como una variable de riesgo del microsistema. Algunas investigaciones reportan que la violencia en la pareja tiene relación con los estilos disciplinarios violentos (Frías y McCloskey, 1998; Youssef, Attia y Kamel, 1998; Margolin et al., 2003). Gaxiola y Frías (2005) mencionaron que la relación entre la violencia doméstica y los estilos de crianza violentos, puede ser por el estrés que provoca ser receptora de violencia por parte de la pareja; algunos autores señalan que dichos efectos son similares al estrés postraumático (Chemtob y Carlson, 2004).

En el ontosistema puede encontrarse como variable de riesgo la depresión. La depresión materna está asociada alta y significativamente a las estrategias de crianza empleadas con los hijos (Hammen, 2003). Los resultados de algunas investigaciones señalan que las madres depresivas presentan comportamientos más irritables u hostiles con sus hijos, además sus actos son menos predecibles y tienden a despreocuparse de sus hijos (Langrock Compas, Keller, Merchant, y Copeland, 2002; Lovejoy, Graczyk, O'Hare y Neuman, 2000; Lyons-Ruth, Wolfe y Lyubchik, 2000). La relación entre la depresión y los estilos disciplinarios violentos ya fue establecida para una muestra con características similares a las del presente estudio (Frías, 2002).

Atendiendo a lo señalado anteriormente en el aspecto metodológico, serán probadas en un modelo estructural, las relaciones directas e indirectas entre variables protectoras y de riesgo del ontosistema, del microsistema, del exosistema y del macrosistema, las cuales son señaladas por la literatura como variables relevantes de protección y de riesgo relacionadas con los estilos de crianza, y también, con la probabilidad para el maltrato infantil. El presente estudio es la continuación de una línea de investigación, donde en un primer momento, se han estudiado los estilos de crianza de las madres de familia; sin embargo, en futuras investigaciones se requiere de la evaluación de 
los factores asociados a los estilos de crianza de los padres.

Debido a que los factores protectores y los factores de riesgo pueden encontrarse en múltiples dominios, es necesario la construcción de modelos integradores que midan en su amplitud contextual dichos factores (Luthar, Cicchetti y Becker, 2000), empleando por ejemplo los modelos de ecuaciones estructurales para el análisis de las relaciones directas e indirectas entre las variables (Bentler, 2006; Serbin y Karp, 2004).

Por tal motivo, el objetivo de la presente investigación fue identificar los factores que protegen a las madres, frente a la probabilidad de utilizar con sus hijos los estilos de crianza autoritarios, ante la presencia de riesgos específicos como la inseguridad del vecindario, la violencia de pareja y la depresión.

\section{Método}

\section{Participantes}

Fueron entrevistadas 182 madres de familia en situaciones de riesgo para conformar un muestreo deliberado por heterogeneidad (Cook y Campbell, 1979), que cumplieron con el requisito de tener una relación estable con su pareja durante el último año, y con al menos un hijo de entre 6 y 12 años.

Los factores de riesgo que se tomaron en cuenta para la conformación de la muestra fueron aquellos que de acuerdo con la literatura probabilizan el riesgo de utilizar tácticas disciplinarias violentas con sus propios hijos. Éstos fueron: a) Madres que acudían a buscar atención médica en los hospitales (Lowne y Vega, 2001); b) Madres con niños en escuelas de educación especial (Ateha y Durrant, 2005); c) Madres con número de hijos arriba del promedio para el Estado (Ethier, Couture y Lacharité, 2004); d) Madres con problemas de consumo de alcohol y drogas (Berger, 2004); y, e) Madres con historia de violencia con la pareja (Margolin y Gordis, 2003).

\section{Instrumentos}

Fue conformado un inventario que incluyó preguntas demográficas como la edad de la madre y su estatus matrimonial, el ingreso familiar mensual, el ingreso separado por cada uno de los miembros de la pareja, la ocupación del compañero y el nivel educativo de los padres. La Escala de Prestigio del Trabajo Americano (Stevens y Hoisingnton's, 1987) se utilizó para obtener indicadores del estatus ocupacional de las madres y padres adaptada al contexto mexicano, donde resultaron las categorías de desempleado, peón u obrero especializado y no especializado, empleado administrativo o equivalente, cuadros medios y cuadros altos de la empresa.

Los niveles de depresión fueron evaluados empleando la Escala de Depresión de Hamilton (Hamilton, 1959, Hamilton y Collins, 1981). Las madres encuestadas reportaron el número de veces que tuvieron alguno de los sentimientos enumerados en la escala en las últimas dos semanas. En estudios previos desarrollados en la localidad, se reportaron valores apropiados de confiabilidad y validez; Figueredo et al. (2001) obtuvieron un alfa de .79 y Frías (2002) reportó un alfa de .72.

Los niveles de violencia de la pareja se vaIuaron con la escala de Tácticas de Conflicto de Straus (1990). Dicha escala fue elaborada en los Estados Unidos y traducida al español por expertos. Los reactivos de la escala miden la frecuencia con la que las mujeres recibieron agresiones por parte de sus parejas. Straus reportó un alfa de Cronbach de .78 para toda la escala y Gaxiola y Frías (2005) reportaron un alfa de .91 .

El apoyo de la pareja se midió con una escala elaborada especialmente para el estudio por Gaxiola y Frías (no publicada). Dicha es- 
cala cuenta con 22 reactivos que miden la frecuencia con que la pareja realiza actividades de apoyo. En el estudio piloto del instrumento resultó un alfa de .93 .

Una escala elaborada para la investigación por Gaxiola y Ribes (no publicada) fue utilizada para valorar los estilos de crianza. Dicha escala está conformada por 132 preguntas que pretenden medir los estilos de crianza y su estabilidad de acuerdo con la clasificación de Baumbrind (1967, 1983, 1991). La escala cuenta con un total de 132 preguntas que evalúan los estilos autoritario, autoritativo y permisivo en tres momentos: pasado (hace un año), presente (desde hace un mes), futuro (a partir del siguiente evento). En el piloteo de la escala resultó un alfa de .77.

Para medir las características de inseguridad del vecindario fue aplicada una escala elaborada por Frías-Armenta, López-Escobar y Díaz-Méndez (2003), la cual reportó un alpha de .85. La escala está compuesta por 9 preguntas donde los encuestados califican diversas características del lugar donde viven. También se aplicaron seis preguntas adicionales, elaboradas por las mismas autoras para evaluar la cohesión social del vecindario, la cual presentó un alfa de .85 en el estudio piloto.

La autoestima fue medida empleando 13 preguntas del inventario de Harterard compilado en el proyecto DICA-R-7.3 de la Washington University School of Medicine (Reich, 1992) que expresaron la opinión que las encuestadas tuvieron sobre sí mismas.

El apoyo social se midió con un cuestionario de 18 preguntas conformado con dos escalas incluidas en el proyecto DICA-R-7.3 de la Washington University School of Medicine (Reich, 1992). Una de ellas mide la frecuencia de las interacciones de los familiares y amigos y la otra mide la frecuencia del apoyo social emocional e instrumental de los mismos. En el estudio piloto resultó un alfa de .82 para todo el cuestionario en su conjunto.
El factor $\mathrm{K}$ fue evaluado con el instrumento desarrollado por Figueredo et al. (2005) denominado mini $\mathrm{K}$, el cual en un estudio piloto llevado a cabo en Tucson, Arizona, y en Hermosillo, Sonora, obtuvo un alfa de Cronbach arriba de .60.

Por último, la calidad de vida se midió con la versión breve del Inventario de Calidad de Vida (WHOQOL-Bref), el cual presenta 25 preguntas en cuatro dominios: salud física, salud psicológica, relaciones sociales y aspectos ambientales. Carr (2003) reportó un alfa de Cronbach para la escala de .66 a .97.

\section{Procedimiento}

Las madres de familia fueron entrevistadas en sus casas, en las salas de espera de hospitales públicos y de instituciones que atienden violencia intrafamiliar, en salones de clase de primarias de la periferia de la ciudad, y en cuartos proporcionados por una institución que atiende a mujeres en rehabilitación de abuso de alcohol y drogas. Todos los directores de los organismos fueron contactados antes del estudio para explicarles brevemente el proyecto y entregarles una solicitud formal, donde estaban especificados los objetivos y las características generales de la investigación, además fue anexada una copia de la entrevista. Las madres fueron entrevistadas previo consentimiento informado por estudiantes de la escuela de Psicología de la Universidad de Sonora y por pasantes de la misma escuela entrenados en el levantamiento de datos. La entrevista duró aproximadamente 75 minutos.

\section{Análisis de datos}

Primeramente se obtuvieron estadísticas univariadas, medias y desviaciones estándar para las variables continuas y frecuencias para las variables categóricas. Las variables negativas fueron multiplicadas por - 1 para estandarizarlas con las variables positivas; éstas fueron las variables depresión, violencia de pareja, 
historia de abuso, estilo de crianza autoritario, estilo de crianza permisivo y abuso infantil.

Después fueron elaborados índices promediando las variables de las escalas. Se obtuvieron las alfas de cada uno de los índices conformados, y posteriormente los datos fueron analizados utilizando un modelo de ecuaciones estructurales (Bentler, 2006). Cada uno de los factores teóricos propuestos fue probado con sus indicadores, así como sus interrelaciones en modelos de correlación y regresión, también se eliminaron las relaciones no significativas entre los factores y las variables indicadoras. Posteriormente, fue probado el modelo de relaciones estructurales. El macrosistema fue eliminado del modelo al no obtenerse los datos de los diversos estratos socioeconómicos para realizar las comparaciones.

\section{Resultados}

\section{Variables demográficas}

La edad promedio de las mujeres fue de 34.8 años (desviación estándar 6.3), el núme- ro de hijos tuvo una media de 2.6 (desviación estándar 1.0) y los años de escolaridad de las madres tuvieron una media de 8.6 (desviación estándar 6.6), lo que equivale en promedio a segundo grado de secundaria; la media de convivencia con la pareja fue de 12.4 años (desviación estándar 5.1). La Tabla 1 presenta las características demográficas de la muestra.

Se obtuvieron alfas para cada una de las escalas de la investigación las cuales son presentadas en la Tabla 2. Todas las alfas resultaron superiores a .60 por lo cual fueron consideradas aceptables.

La Tabla 3 muestra las correlaciones de Pearson de donde se obtuvieron las variables y el factor protector del modelo estructural. Todas las correlaciones fueron significativas a $\mathrm{p}<.0001$. Se conformaron de manera significativa los constructos exosistema, microsistema, ontosistema, crianza y factor protector.

\section{Tabla 1}

Variables Demográficas de la Muestra

\begin{tabular}{lcr}
\hline Variable & $\mathrm{M}$ & D. E. \\
\hline Edad & $(\mathrm{N}=182)$ & 6.3 \\
Tiempo de vivir con su pareja & 34.8 & 5.1 \\
Ingreso de pareja* $^{*}$ & 12.4 & 7405.8 \\
Ingreso propio* $^{*}$ & 6058.3 & 3539.1 \\
Ingreso de algún otro tipo* $^{*}$ & 1609.1 & 649.6 \\
Ingreso familiar* & 137.9 & 8216.6 \\
Años de escolaridad de pareja & 7495.5 & 7.1 \\
Años de escolaridad propia & 8.5 & 6.6 \\
Número de hijos & 8.6 & 1.0 \\
\hline
\end{tabular}

*Pesos mexicanos al mes 


\section{Tabla 2}

Alfas de las Escalas Empleadas en la Investigación

\begin{tabular}{lc}
\hline Escala & Alfa de Cronbach \\
\hline Apoyo social familiares y amigos & 0.79 \\
Cohesión del vecindario & 0.85 \\
Seguridad del vecindario & 0.92 \\
Apoyo de pareja & 0.93 \\
Violencia de pareja & 0.93 \\
Estilo autoritativo & 0.93 \\
Estilo autoritario & 0.78 \\
Estilo permisivo & 0.94 \\
Crianza & 0.84 \\
Factor K & 0.82 \\
Autoestima & 0.84 \\
Depresión & 0.97 \\
Calidad de vida & 0.90 \\
\hline
\end{tabular}

\section{Tabla 3}

Correlaciones de Pearson de las variables utilizadas en el modelo estructural y del factor protector

\begin{tabular}{|c|c|c|c|c|c|c|}
\hline Variable & $\mathrm{N}$ & Exosistema & Microsistema & Ontosistema & Crianza & $\begin{array}{l}\text { Factor } \\
\text { Protector }\end{array}$ \\
\hline Apoyo social & 174 & 0.72 & & & & \\
\hline Cohesión del vecindario & 180 & 0.60 & & & & \\
\hline Seguridad del vecindario & 182 & 0.61 & & & & \\
\hline Apoyo de pareja & 181 & & 0.73 & & & \\
\hline \multicolumn{7}{|l|}{ Apoyo social de } \\
\hline familiares y amigos & 182 & & 0.71 & & & \\
\hline Violencia de pareja & 181 & & 0.77 & & & \\
\hline Autoestima & 182 & & & 0.75 & & \\
\hline Calidad de vida & 182 & & & 0.76 & & \\
\hline Depresión & 181 & & & 0.64 & & \\
\hline Estilo autoritativo & 181 & & & & 0.82 & \\
\hline Autoritario & 181 & & & & -0.80 & \\
\hline Permisivo & 181 & & & & -0.84 & \\
\hline Exosistema & 182 & & & & & 0.59 \\
\hline Microsistema & 182 & & & & & 0.79 \\
\hline Ontosistema & 182 & & & & & 0.79 \\
\hline
\end{tabular}




\section{Modelo estructural}

La Figura 1 muestra los resultados del modelo estructural. Los indicadores de bondad de ajuste del modelo mostraron su pertinencia al obtener valores cercanos a 1, además, resultó una X2 de 15.8 basada en $13 \mathrm{GL}$, con una probabilidad asociada de 0.25 , lo cual permite concluir que el modelo teórico evaluado no es significativamente diferente del modelo saturado. La R2 del modelo con respecto a los estilos de crianza fue de .05 .
En el modelo presentado el factor $\mathrm{K}$ predijo a los factores protectores (.65), construidos a partir de variables del exosistema, microsistema y ontosistema. Dicha relación implica que a mayor inversión paterna aumentan los factores protectores. Estos datos muestran que las madres altas en el factor $\mathrm{K}$ tienden a interactuar con variables protectoras del exosistema, el microsistema y el ontosistema, las cuales pueden protegerlas de los efectos adversos de las variables de riesgo, encontradas también

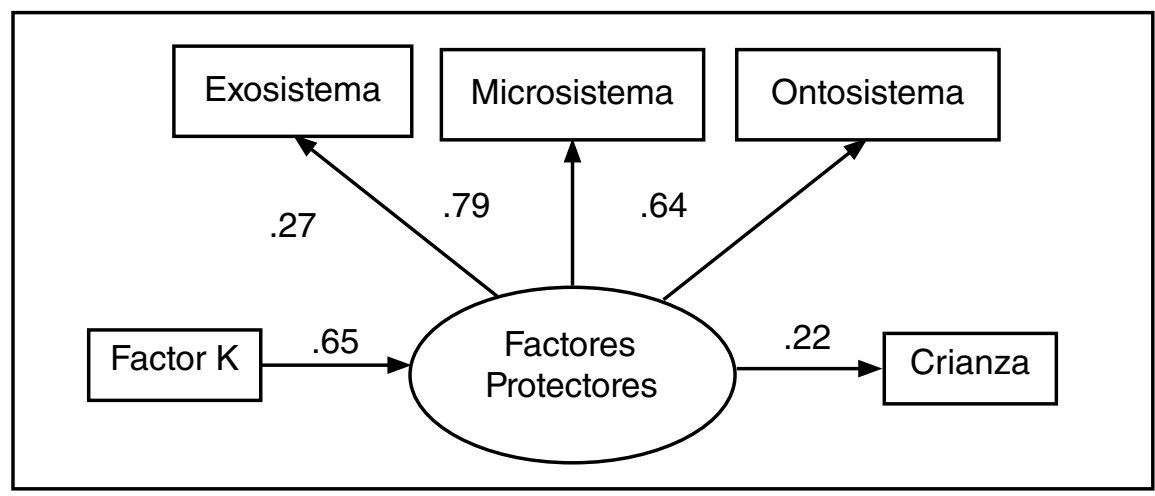

$X^{2}=14.65$ G. de L. $p=0.01 \quad \mathrm{BBNFI}=.90 \quad \mathrm{BBNNFI}=.86 \mathrm{GFI}=.96 \quad \mathrm{RMSEA}=.04 R^{2}=.05$

Figura 1. Modelo estructural de los factores protectores del exosistema, microsistema, ontosistema y la crianza.

\section{Discusión}

El presente estudio identificó algunos de los factores protectores presentes en la ecología de las madres encuestadas que las protegen de las consecuencias negativas de las variables de riesgo en la crianza de sus hijos. Las variables protectoras de los sistemas evaluados fueron, en el exosistema, el apoyo social de los vecinos y la cohesión social; en el microsistema, el apoyo de pareja y el apoyo social de familiares y amigos; y del ontosistema, la calidad de vida y la autoestima. Las variables de riesgo fueron del exosistema la inseguridad del vecindario, del microsistema la violencia de pareja, y del ontosistema la depresión. en dichos sistemas. Así, a mayor factor K, se obtiene mayor beneficio de los apoyos sociales disponibles desde la pareja, los familiares y amigos y los vecinos, lo cual avala el principio que indica una relación positiva entre el factor $\mathrm{K}$ y el comportamiento prosocial (Figueredo et al., 2005). El apoyo social funciona indirectamente, proporcionando aceptación emocional que puede proteger del impacto del estrés sobre los padres (Simons, Lorenz, Wu y Conger, 1993). Garbarino y Kostelny (1995) mencionan que el apoyo social sirve para que los padres se sientan conectados a su comunidad, y promueve que se utilicen estrategias disciplinarias no punitivas al tener posibilidad de recibir apoyo instrumental (por ejemplo dinero), apoyo emocional o información sobre estrategias de crian- 
za (Cochran y Niego, 1995). Por otra parte, los apoyos recibidos por las madres de familia extendida y de los amigos, puede incrementar la probabilidad del empleo de las estrategias de crianza de tipo autoritativo. En diversas investigaciones la explicación de la relación entre el apoyo social recibido y los estilos de crianza, está en función del impacto del apoyo social en la obtención de recursos materiales y emocionales para las madres de familia (Bowen, Bowen y Ware, 2002; Garbarino, Kostelny y Barry, 2002; Korbin, 2003), que afectan su bienestar familiar general (Armstrong, Birnie-Leftcovitch y Ungar, 2005).

El apoyo de la pareja resultó ser una variable protectora en el microsistema. Para Belsky (1984) y Belsky y Vondra (1989), el apoyo social de la pareja constituye el principal sistema de apoyo con respecto a los determinantes del comportamiento de crianza en los padres. De acuerdo con dichos autores, la calidad de las relaciones de pareja probablemente establece un clima de acuerdo y cooperación, lo cual puede ser la base para explicar que en este estudio, las relaciones de pareja predijeron a los estilos de crianza con tendencias autoritativas. Los resultados encontrados fortalecen la teoría de Belsky (1984) sobre los determinantes familiares de los estilos de crianza, al aportar datos a favor del papel protector del apoyo social de la pareja. DeLongis, Capreol, Holtzman, O'Brien, y Campbell (2004) encontraron que el apoyo de la pareja influye en el afecto positivo de sus miembros y esto a su vez afecta su bienestar, por su parte Purdom, Lucas y Miller (2006) señalaron que al incrementarse el apoyo del esposo la satisfacción de la relación puede incrementarse.

Otra de las variables indicadoras del exosistema señala que los efectos positivos de la cohesión social del vecindario donde viven las madres, pueden servir como un factor protector de los riesgos de la historia de abuso, lo cual está de acuerdo con los modelos de eficacia colectiva (Sampson Raudenbush y Earls, 1997;
Boyce et al., 1998), los cuales establecen que la cohesión social de los vecindarios influye en las estrategias utilizadas por los padres en el contexto familiar. Silk et al. (2004) reportaron que la cohesión social del vecindario es un factor protector del los estilos maternales hostiles. Así, a mayor cohesión social, mayor probabilidad de tener características positivas en los estilos de crianza, establecidos en esta investigación por el estilo de crianza autoritativo.

La autoestima y la calidad de vida, como variables indicadoras del ontosistema, también se vieron afectadas por el nivel alto del factor $\mathrm{K}$. Esto puede explicarse debido a que el factor $\mathrm{K}$ agrupa comportamientos prosociales, de pensamiento a largo plazo e inversión materna, que elevan la calidad de vida y la autoestima de las personas. Es probable que las personas altas en el factor $\mathrm{K}$ se adapten de una manera más flexible a las exigencias establecidas por los riesgos ambientales, al emplear los recursos sociales y materiales de que disponen en sus entornos inmediato; además, los grados de satisfacción personal pueden establecer un clima de seguridad, control y apoyo sobre los efectos adversos de los entornos de riesgo.

A partir de lo discutido anteriormente, es posible concluir que la inseguridad del vecindario en el exosistema, la violencia de pareja del microsistema y la depresión en el ontosistema, establecen riesgos para la crianza que pueden ser amortiguados por las variables protectoras disponibles en las madres con alto nivel del factor K. De esta manera, y de acuerdo con la teoría bioecológica, el factor $\mathrm{K}$ se constituye en un factor proximal debido a que posibilita la transformación de los genotipos en fenotipos.

$\mathrm{La}$ influencia del factor $\mathrm{K}$ en las variables de protección, pone de manifiesto la importancia de incluir el estudio de las variables de índole genética en los procesos de protección de los factores de riesgo, acordes a las últimas tendencias en los estudios del fenómeno de resiliencia (Rutter, 2007). Además, justifica el ampliar la teoría ecológica de Bronfenbrenner 
(1979) a la teoría bioecológica (Bronfenbrenner y Ceci, 1994), para el análisis de los procesos del desarrollo humano.

Como se esperaba, los factores protectores tuvieron una relación positiva con los estilos de crianza (lambda $=.22$ ), lo que señala que a mayores factores protectores, mejor estilo de crianza, caracterizado en la investigación como estilo autoritativo. De este modo, fomentar las relaciones de apoyo social desde el nivel del vecindario, los familiares y amigos, así como de la pareja en las madres en riesgo, puede entonces, incidir en la utilización de estrategias de crianza autoritativas con los hijos. De este modo, al elaborar acciones para fomentar la calidad de vida y la autoestima de las madres en riesgo, puede afectar positivamente las relaciones entre ellas y sus hijos.

Debido a las características de la población donde fue realizado el estudio, los resultados presentados pueden ser un punto de partida para la investigación y análisis de poblaciones similares a las de la muestra. Los hallazgos también pueden servir de base para tomar en consideración las variables del modelo, tanto en estudios preventivos como de rehabilitación en madres de familia que presenten riesgos similares a los de la muestra. Empleando el modelo ecológico Corcoran y Nichols-Casebolt (2004), proponen elaborar programas que atiendan los factores de riesgo de los diversos sistemas y fortalezcan los factores protectores que rodean a las personas, lo cual puede ser el marco general en el cual pueden desarrollarse estas estrategias.

El modelo presentado explica un 5 por ciento de la crianza, lo cual implica que existen otras variables que explican el fenómeno que es necesario tomar en cuenta en estudios futuros sobre el tema. Se requiere investigar también cómo algunos eventos displacenteros y potencialmente peligrosos pueden fortalecer a los individuos frente a eventos similares en un proceso de inoculación al estrés (Rutter, 1985). Rutter (1990) señala que es importante identi- ficar los factores de riesgo y protección, en tanto éstos permiten predecir resultados negativos 0 positivos en el proceso de desarrollo de las personas. Es a partir de la medición de las consecuencias de diversas variables en el desarrollo de las personas en contextos específicos, como se pueden identificar los otros factores protectores no contemplados en el modelo. También será de utilidad de acuerdo con el mismo autor, la identificación de los mecanismos y procesos que llevan a convertir a unos factores en protectores y a otros de riesgo.

Entre las limitaciones del estudio se puede mencionar que es un estudio retrospectivo con probables olvidos de información; también los datos pueden ser afectados por las circunstancias presentes en la vida de las madres encuestadas, o bien por el contexto mismo de la entrevista. Otra de las limitaciones es que la información fue obtenida por medio del reporte verbal de las madres. Por tal motivo, se propone que en los estudios posteriores de los estilos de crianza que sean medidos con registros observacionales de las interacciones madres-hijos, al respecto Bennett, Sullivan y Lewis (2006), reportaron la posibilidad de identificar a las madres que utilizan estrategias de crianza hostiles empleando interacciones de enseñanza de armado de rompecabezas con los hijos. También es necesario incluir en los estudios futuros a los padres de familia. 


\section{Referencias}

Armstrong. M. I., Birnie-Leftcovitch \& Ungar, M. T. (2005). Pathways between social support, family well being, quality of parenting, and child resilience: what we know. Journal of Child and Family Studies, 14, 269-281.

Ateah, C.A. \& Durrant, J. E. (2005). Maternal use of physical punishment in response to child misbehavior: implications for child abuse prevention. Child Abuse y Neglect, 29, 169-185.

Baumrind, D. (1967). Child care practices anteceding three patterns of preschool behavior. Genetic Psychology Monographs, 75, 43-88.

Baumrind, D. (1991). Parenting styles and adolescent development. In J. Brooks-Gunn, R. Lerner \& A. C. Petersen (Eds.). The encyclopedia of adolescence (pp. 746-758). New York: Garland.

Baumrind, D. (1983). Rejoinder to Lewis's reinterpretation of parental firm control effects: Are authoritative families really harmonious? Psychological Bulletin, 94, 132-142.

Belsky, J. (1980). Child maltreatment an ecological integration. American Psychologist, 35, 320- 335.

Belsky, J. (1984). The determinants of parenting: A process model. Child Development, 55, 83-96.

Belsky, J. \& Vondra, J. (1989). Lessons from child abuse: The determinants of parenting. In D. Cicchetti \& V. Carlson (Eds.), Child maltreatment: Theory and research on consequences of child abuse and neglect (pp. 153-202). New York: Cambridge University Press.

Bennett, D. S., Sullivan, M. W. \& Lewis, M. (2006). Relations of parental report and observation of parenting to maltreatment history. Child Maltreatment, 11, 63-75.

Bentler, P. M. (2006). EQS 6 Structural Equations Program Manual. Encino, CA: Mulivariate Software Inc.

Berger, L. M. (2004). Income, family structure, and child maltreatment risk. Children and Youth Services Review, 8, 725-748.

Bowen, N. K., Bowen, G. L. \& Ware, W. B. (2002). Neighborhood social disorganization, families, and the educational behavior of adolescents. Journal of Adolescent Research, 17, 468-490.

Boyce, W. T., Frank, E., Jensen, P. S., Kessler, R. C., Nelson, C. A., Steinberg, L., et al. (1998). Social context in developmental psychopathology: Recommendations for future research from the MacArthur Network on Psychopathology and Development. Development and Psychopathology, 10, 143-164.

Bronfenbrenner, U. (1979). La Ecología del Desarrollo Humano. Madrid: Paidós.

Bronfenbrenner, U. \& Ceci, S. J. (1994). Nature-nuture reconceptualized in developmental perspective: A bioecological model. Psychological Review, 101, 568-586.

Carlson, B. E., McNutt, L. \& Choi, D. H. (2003). Childhood and adult abuse among women in primary health care, effects on mental health. Journal of Interpersonal Violence, 18, 924-941.

Carr, A. (2003). Adult measures of quality of life. Artrithis y Rheumatism, 49, s113-s133.

Chemtob, C. M. \& Carlson, J. G. (2004). Psychological Effects of Domestic Violence on Children and Their Mothers. International Journal of Stress Management, 11, 209-226.

Cicchetti, D. \& Rogosch, F. A. (1997). The role of self-organization in the promotion of resilience in maltreated children. Development and Psychopathology, 9, 797-815.

Cochran, M. \& Niego, S. (1995). Parenting and social networks. In M. H. Bornstein (Ed.). Handbook of parenting: Vol. 3. Status and social conditions of parenting (pp. 393-418). Mahwah, NJ: Erlbaum.

Corcoran, J. \& Nichols-Casebolt, A. (2004). Risk and resilience ecological framework for assessment and goal formulation. Child and Adolescent Social Work Journal, 3, 211-235.

Cook, T. D. \& Campbell, D. T. (1979). Quasi-Experimental Design y Analysis Issues for Field Settings. Houghton Mifflin Company.

Crouch, J. L. \& Behl, L. E. (2001). Relationships among parental beliefs in corporal punishment, reported stress, and physical child abuse potential. Child Abuse and Neglect, 3, 413-419.
Darling, N. \& Steinberg, L. (1993). Parenting style as a context: an integrative model. Psychological Bulletin, 113, 487-496.

Dehle, C., Larsen, D. \& Landers, J. E. (2001). Social support in marriage. The American Journal of Family Therapy, 29, 307-324.

DeLongis, A., Capreol, M., Holtzman, S., O’Brien, T. \& Campbell, J. (2004). Social support and social strain among husbands and wives: a multilevel analysis. Journal of Family Psychology, 18, 470-479.

DuMont, K. A., Widom, C. S. \& Czaja, S. J. (2007). Predictors of resilience in abused and neglected children grown-up: The role of individual and neighborhood characteristics. Child Abuse and Neglect, 31, pp. 255-274.

Englund, M. M., Luckner, A. E., Whaley, G. J. \& Egeland, B. (2004). Children's Achievement in Early Elementary School: Longitudinal Effects of Parental Involvement, Expectations, and Quality of Assistance. Journal of Educational Psychology, 4, 723-730.

Emery, R. E. \& Laumann-Billings, L. (1998). An overview of the nature, causes, and consequences of abusive family relationships. American Psychologist, 2, 121- 135.

Ethier, L. S., Couture, G. \& Lacharité, C. (2004). Risk factors associated with the cronicity of high potencial for child abuse and neglect. Journal of Family Violence, 19, 13- 24.

Figueredo, A. J., Corral, V., Frías, M., Bachar, K. J., White, J, McNeill, P. L., et al. (2001). Blood, solidarity, status and honor. The sexual balance of power and spousal abuse in Sonora, Mexico. Evolution and Human Behavior, 22, 295-328.

Figueredo, A. J., Vázquez, G., Brunbach, B. H., Shneider, S., Sefcek, J. A., Tal, I. R., et al. (2005). Consiliencia y la teoría de historia de vida: de los genes al cerebro y a la estrategia reproductiva. In M. Frías y V. Corral (Eds.). Niñez, Adolescencia y Problemas Sociales (pp. 2963), CONACYT y Unison, México.

Flouri, E. (2005). Women's Psychological Distress in Midadulthood: The Role of Childhood Parenting Experiences. European Psychologist, 2, 116-123.

Frías-Armenta, M., López-Escobar, A. E. y Díaz-Méndez, S. G. (2003). Predictores de la conducta antisocial juvenil: un modelo ecológico. Estudios de Psicología, 8, 15-24.

Frías, M. (2002). Long- term effects of child punishment on Mexican women: a structural model. Child Abuse and Neglect, 26, 371-386.

Frías, M. \& McCloskey, L. A. (1998). Determinants of harsh parenting in Mexico. Journal of Abnormal Child Psychology, 26, 129-139.

Furnham, A. y Cheng, H. (2000). Lay theories of happiness. Journal of Happiness Studies, 1, 227-246.

Garbarino, J. \& Kostelny, K. (1995). Parenting and public policy. In M. $\mathrm{H}$. Bornstein (Ed.). Handbook of parenting: Vol. 3. Status and social conditions of parenting (pp. 419-436). Mahwah, NJ: Erlbaum.

Garbarino, J., Kostelny, K. \& Barry, F. (2002). Neighorhood-based programs. In Trickett, P. K. \& Schellenbach, C. J. (Eds.). Violence Against Children in the Family and the Community (pp. 287-314). Washington DC: American Psychological Association.

Gaxiola, R. J. y Frías, M. (2005). Las consecuencias del maltrato infantil: un estudio con madres mexicanas. Revista Mexicana de Psicología, 22, 363-374.

Gershoff, E. T. (2002). Corporal punishment by parents and associated child behaviors and experiences: a meta-analytic and theoretical review. Psychological Bulletin, 128, 539-579.

Hammen, C. (2003). Interpersonal stress and depression in women. Journal of Affective Disorders, 74, 49-57.

Hamilton, M. (1959). The assessment of anxiety states by ratings. British Journal of Medical Psychology, 32, 50-55.

Hamilton, C. J. \& Collins, J. J. (1981). The role of alcohol in wife beating and child abuse: A review of the literature. In J. J. Collins (Ed.). Drinking and Crime: Perspectives on the Relationships between Alcohol Consumption and Criminal Behavior, pp. 253-287. New York: Guilford Press.

Hyland, M. E. (1998). Defining and measuring quality of life in medicine. JAMA, 59-65. 
Korbin, J. E. (2003). Neighborhood and community connectedness in child maltreatment research. Child Abuse and Neglect, 27, 137-140.

Langrock, A. M., Compas, B. E., Keller, G., Merchant, M. J. \& Copeland, M. E. (2002). Coping with the stress of parental depression: Parent's reports of children's coping, emotional, and behavioral problems. Journal of Clinical and Child Psychology, 31, 312-324.

Louis, V. V. \& Zhao, S. (2002). Effects of family structure, family SES, and adulthood experiences on life satisfaction. Journal of Family Issues, 23, 986-1005.

Lovejoy, M. C., Graczyk, P. A., O'Hare, E. \& Neuman, G. (2000). Maternal depression and parenting behavior: A meta-analytic review. Clinical Psychology Review, 20, 561- 592 .

Lowne, E. A. \& Vega, W. A. (2001). Intimate partner violence and health: self-assessed health, chronic health, and somatic symptoms among Mexican American Women. Psychosomatic Medicine, 63, 3, 352360.

Luthar, S. S., Cicchetti, D. \& Becker, B. (2000). The construct of resilience: A critical evaluation and guidelines for future work. Child Development, 71, 543-562.

Lyons-Ruth, K., Wolfe, R. \& Lyubchik, A. (2000). Depression and the parenting of young children: Making the case for early preventive mental health services. Harvard Review of Psychiatry, 8, 148- 153.

Maccoby, E. E. \& Martin, J. A. (1983). Socialization in the context of the family: parent-child interaction. In P. H. Mussen (Series Ed.) y E. M. Hetherington (Vol. Ed.), Handbook of child psychology: Vol. 4. Socialization, personality, and social development (4th ed., pp. 1-101) New York: Wiley.

Mann, M., Hosman, C. M. H., Schaalma, H. P. \& De Vries, N. K. (2004) Self-esteem in a broad-spectrum approach for mental health promotion. Health Education Research. 19, 357-372.

Margolin, G. \& Gordis, E. B. (2003). Co-Ocurrence between marital aggression and parents' child abuse potential: The impact of cumulative stress. Violence and Victims, 3, 243-258.

Margolin, G., Gordis, E. B., Medina, A. M. \& Oliver, P. H. (2003). The cooccurrence of husband-to-wife aggression, family-of-origin aggression, and child abuse potential in a community sample. Journal of Interpersonal Violence, 18, 413-440.

Muller, R. T. \& Lemieux, K. E. (2000). Social support, attachment, and psychopathology in high risk formerly maltreated adults. Child Abuse and Neglect, 24, 883-900.

Munist, M., Santos, H., Kotliarenco, M. A., Suárez-Ojeda, E. N., Infante, F. \& Grotberg, E. (1998). Manual de identificación y promoción de la resiliencia en niños y adolescentes. Organización Panamericana de la Salud y Organización Mundial de la Salud. Washington, D.C.

Purdom, C. L., Lucas, J. L. \& Miller, K. S. (2006). Couple type, parental status, and the mediating impact of social support. North American Journal of Psychology, 8, 1-12.

Reich, W. (1992). Diagnostic Interview for Children and Adolescents- Re- vised (DICA-R-7.3). Washington University School of Medicine, Division of Child Psychiatry.

Rutter, M. (1990). Psychosocial resilience and protective factors mechanisms. In Rolf, J., Masten, A. S., Cicchetti, D., Nuechterlein, K. H. \& Weintraub, S. (Eds.). Risk and Protective Factors in the Development of Psychopathology (pp.181-214), Cambridge: Cambridge University.

Rutter, M. (2007). Resilience, competence, and coping. Child Abuse y Neglect, 3, 205-209.

Rutter, M. (1999). Resilience concepts and findings: implications for family therapy. Journal of Family Therapy, 21, 119-144.

Rutter, M. (1985). Resilience in the face of adversity: protective factors and resistance to psychiatric disorder. British Journal of Psychiatry, $147,598-611$.

Sampson, R., Raudenbush, S. \& Earls, F. (1997). Neighborhoods and violent crime: A multilevel study of collective efficacy. Science, 277 , 918-924.

Serbin, L. A. \& Karp, J. (2004). The intergenerational transfer of psychosocial risk: mediators of vulnerability and resilience. Annual Review of Psychology, 55, 333-363.

Silk, J. S., Sessa, F. M., Morris, A. S., Steinberg, L. \& Avenevoli, S. (2004). Neighborhood cohesion as a buffer against hostile maternal parenting. Journal of Family Psychology, 18, 135-146.

Simons, R. L., Lorenz, F. O., Wu, Ch. \& Conger, R. D. (1993). Social network and marital support as moderators of the impact of stress and depression on parental behavior. Developmental Psychology, 29, 368-381.

Stevens, G. \& Hosingnton, E. (1987). Occupational prestige and the 1980 US labor force. Social Science Research, 16, 74-105.

Straus, M. A. (1990). The Conflict Tactics Scale and its critics: An evaluation and new data on validity and reliability. In M. A. Straus \& R. J. Gelles. (Eds.). Physical Violence in American Families: Risk factors and adaptations to violence in 8,145 families. New Brunswick, $\mathrm{N} \mathrm{J}$ : Transactions Publishers.

Tussaie, K. \& Dyer, J. (2004). Resilience: a historical review of the construct. Holistica Nurse Practice, 18, 3-10.

WHOQOL-Bref. The World Health Organization quality of life assessment (WHOQOL): Development and general psychometric properties. (1998). Social Science y Medicine, 46(12), 1569-1585.

Youssef, R. M., Attia, M. S. \& Kamel, M. I. (1998). Children experiencing violence I: Parental use of corporal punishment. Child Abuse and Neglect, Vol. 22, No.10, 959-973.

Zimmerman, S. L. (2000). Self-esteem, personal control, optimism, extraversion and the subjetive well-being of midwestern university faculty. Dissertation Abstracts International B: Sciences and Engineering, 60 (7-B), 3608. 